\title{
Comment on Half-Integer Quantum Numbers for the Total Angular Momentum of Photons in Light Beams with Finite Lateral Extensions
}

\author{
Manfred Fähnle \\ Max Plank Institute for Intelligent Systems, Stuttgart, Germany \\ Email address: \\ faehnle@is.mpg.de \\ To cite this article: \\ Manfred Fähnle. Comment on Half-Integer Quantum Numbers for the Total Angular Momentum of Photons in Light Beams with Finite Lateral \\ Extensions. American Journal of Modern Physics. Vol. 6, No. 5, 2017, pp. 88-90. doi: 10.11648/j.ajmp.20170605.12
}

Received: July 5, 2017; Accepted: July 20, 2017; Published: August 15, 2017

\begin{abstract}
Recently the spectacular result was derived quantum mechanically that the total angular momentum of photons in light beams with finite lateral extensions can have half-integer quantum numbers. In a circularly polarized Gauss light beam it is half of the spin angular momentum which it would have in a respective infinitely extended wave. In another paper it was shown by a classical calculation that the magnetic moment induced by such a beam in a metal is a factor of two smaller than the one induced by a respective infinitely extended wave. Since the system's angular momentum is proportional to its magnetic moment it could be assumed that the classical result for the magnetic moment reflects the transfer of the total angular momenta of the beam photons to the metal. Here we show that there is no hint that this is indeed the case.
\end{abstract}

Keywords: Light Beams with Finite Lateral Extensions, Total Angular Momentum of Photons, Induced Magnetic Moment in Metal

\section{Introduction}

The angular momentum of photons is heavily discussed in many fields of optics. Starting point thereby often is [1] that Maxwell's equations are invariant under rotations around any direction. This yields the conserved quantity $L_{i}+S_{i}$, where $L_{i}$ and $\mathrm{S}_{\mathrm{i}}$ are the $\mathrm{i}$-components of the orbital and the spin angular momentum, and where the $\mathrm{i}$-axis is parallel to the respective axis of rotation. This quantity is usually conceived as the total angular momentum of photons. The quantum numbers for this total angular momentum are integers. K. E. Ballantine et al. [2] considered the realistic situation in which there is a beam of light with finite lateral extension. Then the system is no longer invariant against rotations around any direction, in the therein mentioned special case of a paraxial beam the reduced symmetry is given by the rotations around the axis of the beam which we denote as z-axis. In such a paraxial beam both spin and orbital angular momentum are valid and independent, which can even be proven for more general cases [3]. Therefore one can discuss a general linear combination

$$
\hat{\mathrm{J}}_{\gamma, \mathrm{z}}=\widehat{\mathrm{L}}_{\mathrm{z}}+\gamma \widehat{\mathrm{S}}_{\mathrm{z}}
$$

where $\widehat{\mathrm{L}}$ and $\widehat{\mathrm{S}}$ are the operators of the orbital and spin angular momentum, respectively. The operator $\hat{\mathbf{J}}_{\gamma, z}$ generates an observable. If the electric field $\mathrm{E}$ of the beam is an eigenfunction, $\left\{\widehat{\mathrm{L}}_{\mathrm{z}}+\gamma \widehat{\mathrm{S}}_{\mathrm{z}}\right\} \mathrm{E}=\mathrm{j}_{\gamma} \mathrm{E}$ the observable can be considered having the meaning of a total angular momentum. In [2] it has been shown that $\gamma$ and $\mathrm{j}_{\gamma}$ are either both integers or half-integers. The first type includes the usual angular momentum operator $\widehat{\mathrm{L}}_{\mathrm{z}}+\widehat{\mathrm{S}}_{\mathrm{z}}$, with eigenvalues which are integer quantum numbers. The second type, typified by $\left\{\widehat{\mathrm{L}}_{\mathrm{z}}+1 / 2 \widehat{\mathrm{S}}_{\mathrm{z}}\right\}$, corresponds to half-integer quantum numbers. The electric fields of circularly polarized Gauss-Laguerre beams [4] are eigenfunctions of $\left\{\widehat{\mathrm{L}}_{\mathrm{z}}+\gamma \widehat{\mathrm{S}}_{\mathrm{z}}\right\}$, and their photons have total angular momenta with half-integer quantum numbers [2]. For a circularly polarized Gauss beam (which is non-helical) the photons have a zero orbital angular momentum and a spin angular momentum $S_{z}$ which is the spin angular momentum $s_{z}$ which they would have in a respective infinitely extended wave, i.e., $S_{z}=S_{z}$. As shown in Ref. [2] the total angular momentum of photons in a 
circularly polarized Gauss beam is $1 / 2 \mathrm{~s}_{\mathrm{z}}$ In Ref. [2] the half-integer quantization of the total angular momentum of beam photons was demonstrated by noise measurements. The findings of Ref. [2] were already used in later papers, for instance on the generation of half-integer orbital angular momentum beams [5] or on fundamentals and applications of conical refraction [6].

In Ref. [7] it was shown by a classical treatment of the inverse Faraday effect (using Newton's equation of motion for the movement of electrons in a classical electric field described by Maxwell's equations) that the magnetic moment $\mathrm{M}$ induced in a metal by a circularly polarized Gauss beam is a factor of two smaller than the one, $\mathrm{M}_{\mathrm{g}}$, induced by an infinitely extended circularly polarized wave. The reduction is caused by a drift current occurring as a result of the finite lateral extension of the Gauss beam, which contributes by $M_{d}=\frac{-M_{g}}{2}$ to the total magnetic moment $M=M_{d}+M_{g}=\frac{M_{g}}{2}$. The effect of an electromagnetic wave on matter reflects the properties of the wave in detail, being more or less a 'fingerprint' of the wave. The system's angular momentum is proportional to its magnetic moment, and the above discussed reduction of the magnetic moment reflects a reduction of the system's angular momentum by a factor of two. The angular momentum of the system is physically generated by a transfer of angular momentum from the beam to the metal. Therefore it could be assumed that the reduction of the system's angular momentum by a factor of two is related to the half-integer quantum number of the total angular momentum of the photons of the beam. We now show that there is no hint that this is actually the case. This is very important for the community working on the total angular momentum of light beams and of their photons, and for the community working on the inverse Faraday effect and on all-optical switching of the magnetization of a material [8].

\section{Orbital and Spin Angular Momentum of a Gauss Beam}

\subsection{Calculation for Classical Fields}

The central idea is that in the classical treatment of Ref. [7] the Gauss beam is not described by a quantum electrodynamics in which the electromagnetic field is built by photons, but it is described by a classical electric field $E(r, t)$. Therefore the electrons of the metal do not 'know' the half-quantization of the photons of the beam, but they 'see' a classical Gauss beam. Therefore we now calculate the spin and orbital angular momentum of this classical field. The angular momentum of an electromagnetic field is [9].

$$
J=\epsilon_{0} \int d^{3} r r \times(E \times B)
$$

This may be subdivided in a spin angular momentum $\mathrm{S}$ and an orbital momentum $\mathrm{L}, \mathrm{J}=\mathrm{L}+\mathrm{S}$. We now calculate $\mathrm{S}$ and $\mathrm{L}$ for a classical circularly polarized Gauss beam. Afterward we consider the total angular momentum of photons in such a beam and explain it in a simple view based on the 'trajectories' of the photons. We consider the electric field E of a circularly polarized Gauss beam with width w and angular frequency $\omega$.

$$
E=\frac{E_{0}}{\sqrt{\left(2 \pi^{3 / 2}\right) w^{3}}} \exp \left\{-r^{2} / w^{2}\right\} \exp \{-i \omega t\}\left(\begin{array}{c}
1 \\
\pm i \\
0
\end{array}\right)
$$

where (+) and (-) stand for left and right circular polarization. This ansatz is normalized in the sense that the integral over the whole space of $E^{\dagger} E$ yields $E_{0}^{2}$. In Ref. [7] an ansatz without the prefactor $\left(\left(2 \pi^{3 / 2}\right) \mathrm{w}^{3}\right)^{-1 / 2}$ is used. We take the normalized form, since we wanted to compare our results with those obtained for an infinitely extended circularly polarized wave

$$
E=\frac{E_{0}}{\sqrt{V}} \exp \{-i \omega t\}\left(\begin{array}{c}
1 \\
\pm i \\
0
\end{array}\right),
$$

which is also normalized in the above sense. Of course the basic result of [7], $M=\frac{\mathrm{M}_{\mathrm{g}}}{2}$, obtained for the non-normalized ansatz, also holds when using the normalized ansatz. For the classical spin angular momentum [10],

$$
\mathrm{S}=\frac{\epsilon_{0}}{2 \mathrm{i} \omega} \int \mathrm{d}^{3} \mathrm{rE} \mathrm{E}^{\dagger} \times \mathrm{E}
$$

we get both for the Gauss beam, $S_{z}$, and for the infinitely extended wave, $s_{z}, S=\left(0,0, S_{z}\right)$ with

$$
\mathrm{S}_{\mathrm{z}}= \pm \epsilon_{0} \mathrm{E}_{0}^{2} / \omega
$$

The same result, $S_{z}=S_{z}$, was also found for the photons in the Gauss beam. For the orbital angular momentum [10]

$$
\mathrm{L}=\frac{\epsilon_{0}}{2 \mathrm{i} \omega} \sum_{\mathrm{i}=\mathrm{x}, \mathrm{y}, \mathrm{z}} \int \mathrm{d}^{3} \mathrm{rE}_{\mathrm{i}}^{*}(\mathrm{r} \times \nabla) \mathrm{E}_{\mathrm{i}}
$$

we get for the infinitely extended wave and for the Gauss beam the result $\mathrm{L}=0$. Remember that the orbital angular momentum of photons in the Gauss beam is also zero, and it is of course also zero for an infinitely extended wave. Altogether this means that the orbital angular momentum of the classical circularly polarized Gauss beam is zero, as the corresponding quantity of the photons in that beam, and that the spin angular momentum $\mathrm{S}_{\mathrm{z}}$ of the beam is equal to the spin angular momentum $s_{z}$ of the infinitely extended wave. However, the total angular momentum of the photons in the beam is $S_{z} / 2$. Therefore there is no hint that the result $\mathrm{M}=\frac{\mathrm{M}_{\mathrm{g}}}{2}$, of the classical calculation can be related to the transfer of the total angular momentum of the photons in the beam. The question therefore arises whether the calculation of the magnetic moment induced by the Gauss beam should be done quantum mechanically instead classically. However, the length- and energy-scales occurring in that problem are within the validity regime of the classical theories (Newton's mechanics and Maxwell's theory), and therefore a quantum mechanical treatment (which would be extremely complicated) is not necessary. There is no doubt that the results of [7] are correct. All this demonstrates that the factors $\frac{1}{2}$ appearing in the theories of Ref. [2] and Ref. [7] do not have the same physical basis. 


\subsection{Comments on the Orbital Angular Momentum of Photons}

In a Gauss beam the z-component $\mathrm{L}_{\mathrm{z}}$ of the orbital angular momentum is a conserved quantity. We now want to explain the result of Ref. [2] that the orbital angular momentum $\mathrm{L}_{z}$ of photons in the non-helical Gauss beam is zero. We explain it from a simple viewpoint which is based on the notion of 'trajectories' of the photons. In principle, the photon state is described by a wavefunction so that one cannot associate a well-defined position to a photon. However, we can construct a wavepacket by a superposition of waves with different wavevectors, and this wavepacket can be made well localized in space. The lateral extension of a helical beam is much larger than the wavelength of the light, and therefore problems arising from the position-momentum uncertainty are not important, and the concept of a photon trajectory seems to be not too bad. For a particle with a trajectory, i.e., for which we can simultaneously define a position $r$ and a linear momentum $p$, the orbital angular momentum is $L=r \times p$. The trajectories of the photons in a Gauss beam are parallel to the beam axis, i.e., $\mathrm{p}=(0,0, \mathrm{p})$. This leads directly to $\mathrm{l}_{\mathrm{z}}=0$. To consider the photon trajectory in a helical beam we note that the trajectory may be conceived as the saddle-point solution of a path-integral representation of the wavemechanics, and this is given by the Eikonal equation [11]. This equation says that the photons choose the path between two points in space for which they have the shortest running time. In a homogeneous medium this is always the straight path (principle of Fermat) [12]. For a helical beam we do not have homogeneity. So the 'shortest' path is not a straight line. When we consider the 'shortest' path as the trajectory of the photon, then the orbital angular momentum $1_{z}$ of the photon is $\hbar \mathrm{m}$, where $\mathrm{m}$ describes the phase winding of the helical wavefront according to the complex exponential $\exp (\mathrm{i} \theta \mathrm{m})$, i.e., we now have a non-vanishing orbital angular momentum of the photon, in contrast to the zero orbital angular momentum of the photon in a non-helical beam.

\section{Conclusion}

A recent quantum mechanical calculation [2] has given the spectacular result that the total angular momentum of photons in circularly polarized light beams can have half-integer quantum numbers. Another recent classical treatment [7] has given an also spectacular result, namely that the magnetic moment induced by such a beam in a metal is a factor of two smaller than the one induced by a respective infinitely extended wave. We demonstrated in the present paper that the two results, which seem to have the same underlying physics, have nothing to do with each other in reality. The result of the quantum mechanical treatment that the photons in a non-helical Gauss beam have zero orbital angular momentum whereas they have a non-zero orbital angular momentum in a helical light beam could be explained from a simple viewpoint based on the notion of photon trajectories. Thereby the meaning of a photon trajectory in a light beam with a width which is much larger than the wavelength of the light is discussed.

\section{Acknowledgements}

The author is indebted to Riccardo Hertel and Paul Eastham for many interesting discussions.

\section{References}

[1] L. Allen, S. Barnett, and M. Padgett, Optical Angular Momentum, Optics \& Optoelectronics (Taylor \& Francis, 2003).

[2] K. E. Ballantine, J. F. Donegan, and P. R. Eastham, Science Advances 2 (2016), 10.1126/sciadv.1501748, http://advances.sciencemag.org/content/2/4/e1501748.full.pdf.

[3] K. Y. Bliokh, J. Dressel, and F. Nori, New Journal of Physics 16, 093037 (2014).

[4] G. F. Calvo, A. Picon, and E. Bagan, Phys. Rev. A 73, 013805 (2006).

[5] A. Turpin, C. Rego, A. Picon, J. S. Roma, and C. Hernan-dez-Garcia, Sci. Rep. 7, 43888 (2017).

[6] A. Turpin, Y. V. Laiko, T. K. Kalkandjev, and J. Mompart, Laser \& Photonics Rev. 10, 750 (2016).

[7] R. Hertel and M. Fähnle, Phys. Rev. B 91, 020411 (2015).

[8] C. D. Stanciu, F. Hansteen, A. V. Kimel, A. Kirilyuk, A. Tsukamoto, A. Itoh, and T. Rasing, Phys. Rev. Lett. 99, 047601 (2007).

[9] D. J Griffiths, Introduction to electrodynamics, 3rd ed. (Pearson/Benjamin Cummings, San Francisco, 2008) "International edition" Cover.

[10] S. M. Barnett, J Mod Opt 57, 1339 (2010), 24808629 [pmid].

[11] D. T. Paris and F. K. Hurd, Basic Electromagnetic Theory (McGraw Hill, 1969).

[12] A. Schuster, An Introduction to the Theory of Optics (Edward Arnold, London, 1904). 